\title{
Synthesis and Characterization of pH Responsive Polyurethane
}

\section{Lucas Polo Fonseca*, Maria Isabel Felisberti.}

\section{Abstract}

Polyurethanes present a great potential in biomedical applications due to their biocompatibility and highly tunable properties such as hydrophilicity and hydrophobicity. Between these properties one that diserves special attention is the $\mathrm{pH}$-responsiviness that allows drug delivery under specific $\mathrm{pH}$ conditions. In this study we present the synthesis and structural characterization of two novel $\mathrm{pH}$ responsive polyurethanes .

\section{Key words:}

Polyurethanes, $\mathrm{pH}$ responsive, biomedical.

\section{Introduction}

Polyurethanes are a versatile class of materials. A rather new application of polyurethanes is its use in drug delivery systems. For such applications the insertion of $\mathrm{pH}$-responsive groups attached to the polyurethane chain can trigger the drug release in a specific regions of the body with a particular $\mathrm{pH} .{ }^{1}$ The use of acid or basic chain extensors that deprotonate or protonate in basic or acid media, respectively, is reported as an strategy for achieving this goal. In this study we present the synthesis and characterization of two novel $\mathrm{pH}$-responsive polyurethanes based on hexamethylene diisocyanate ( $\mathrm{HDI})$ and hydroxyethyl piperazine (HEP), which contains a tertiary amine group, and on $\mathrm{HDI}$ and bis-2.2hydroxymethyl propionic acid (DMPA), which contains carboxylic acid as a pendent group, named PUH and PUD respectively.

\section{Results and Discussion}

The chemical structures of the $\mathrm{pH}$-responsive polyurethanes (PU) are presented in Figure 1.
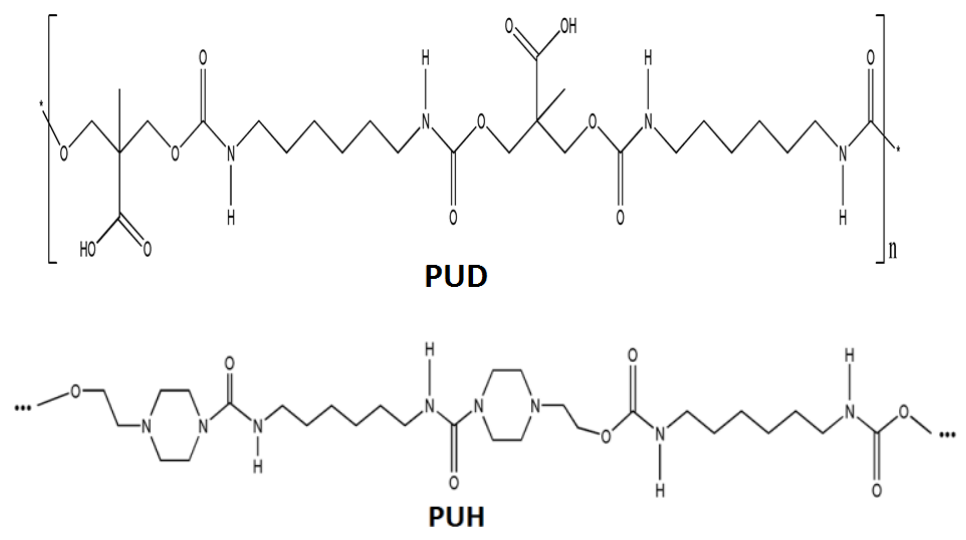

Figure 1: Structures of the $\mathrm{pH}$-responsive polyurethanes PUD and PUH

Fourier transform infrared spectra, Figure 2, show peaks at 1650 and $3300 \mathrm{~cm}^{-1}$ assigned to $\mathrm{C}-\mathrm{N}$ and $\mathrm{N}-\mathrm{H}$ bonds, respectively, demonstrating the formation of the urethane group therefore the success of the polyurethane synthesis. Peaks in the wavenumbers between $1650-1750 \mathrm{~cm}^{-1}$ assigned to the carbonyl bonds are also observed for the polyurethanes.

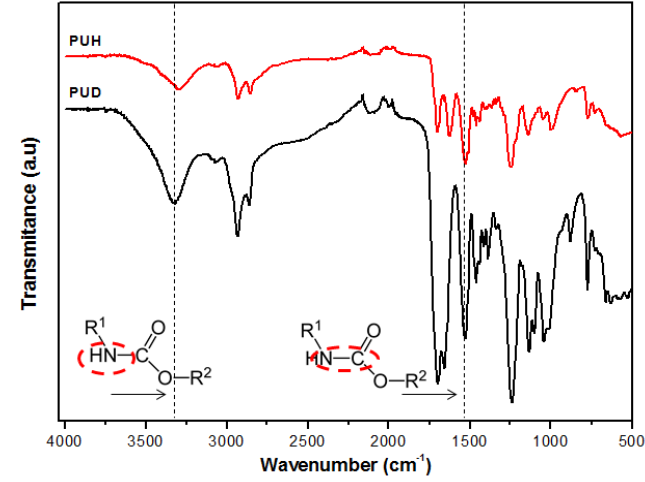

Figure 2: Fourier transform Infrared spectra of PUD and PUH The $\mathrm{pH}$-response of the polyurethanes were demonstrated by solubility tests using buffer solutions at different $\mathrm{pH}$ 's. The images in Figure 3 show that PUD is soluble only in aqueous solutions of $\mathrm{pH} 7$ and 10, due to deprotonation of pendent carboxylic acid group. On the other hand $\mathrm{PUH}$ do not solubilize in any buffer solution probably due to high molar mass $\left(\sim 40 \mathrm{~kg} \mathrm{~mol}^{-1}\right.$, determined by Gel Permeation Chromatography).

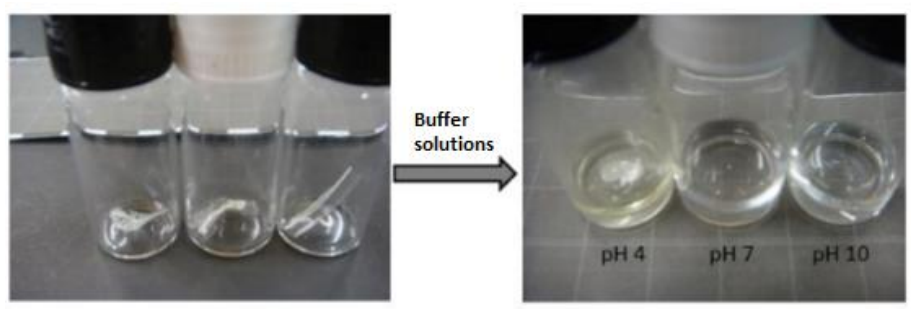

Figure 3: PUD solubility in different $\mathrm{pH}$

\section{Conclusions}

Both PU were successfully synthesized. PUD presented responsive character to $\mathrm{pH}$ being only soluble in $\mathrm{pHs}$ above 7 . The study now is focusing on synthesize triblock copolymers with central block of acid or base PU and lateral blocks of polyethylene glycol (PEG).

\section{Acknowledgement}

The authors acknowledge FAPESP-Brazil (Processes $\mathrm{n}^{\circ}$ 2010/17804-7 and 2015/25406-5) and CNPq-Brazil (Process $n^{\circ} 444392 / 2014-9$ ) for the financial support.

1 - Sardon H., Tan J. P. K., Chan J. M. W., Mantione D., Mecerreyes D., Hedrick J. L. Yang Y. Y.; Macromol. Rapid Commun. 36, 1761-1767, (2015) 